\title{
An Overview of Modeling Yarn's 3D Geometric Configuration
}

\author{
Tianyong Zheng, Jing Wei, Zhengtao Shi, Tingting Li, Zhen Wu \\ School of Textile Engineering, Zhongyuan University of Technology, Zhengzhou, China \\ Email: zty_zzti@126.com
}

Received 14 January 2015; accepted 4 February 2015; published 6 February 2015

Copyright (C) 2015 by authors and Scientific Research Publishing Inc.

This work is licensed under the Creative Commons Attribution International License (CC BY). http://creativecommons.org/licenses/by/4.0/

c) (i) Open Access

\section{Abstract}

Modeling the 3D geometric configuration of yarn is the base of building the 3D fabric structure. The surfaces of the yarns are modeled by many facets. To improve the efficiency of modeling, 3D yarn is usually designed by Bezier curve, spline curve or B-spline curve on the principle of Computer Graphics. The paper reviews different 3D modeling methods for single yarn from the view of cross-section and central line. Some improvements for a better visual effect such as inserting twist and hairiness are illustrated as well. Based on the single yarn, the folded yarn and some fancy yarns can also be modeled in 3D space as long as the cross-sections of the control sections of the component single yarn are determined. Applications of the various 3D yarns are demonstrated. The advantages and the drawbacks of various methods are explained and compared as well. Comparatively speaking, modeling technology based on spline curve is more convenient and flexible, perhaps it is the most popular tool since it is capable of designing different kinds of yarns mentioned above and gets the maximum support from the different developing platforms such as OpenGL, 3DS-MAX, Solid Works CAD, etc.

\section{Keywords}

\section{D Modeling, Yarn, Spline Curve, Computer Graphics}

\section{Introduction}

The geometrical structures of the woven fabrics, knitted fabrics or braided fabrics affect the physical properties of the fabric to a great extent. These fabrics are composed of yarns, therefore, the 3D geometrical configuration of the yarn has to be modeled before the 3D geometrical structures of the fabrics are to be modeled. Various methods were proposed in modeling the 3D yarns and the paper aims to give a complete illustration of how to model a 3D yarn. 


\section{Methodology for Single Yarn}

The configuration of 3D yarn is modeled according to Computer Graphics. Generally speaking, the surface of the $3 \mathrm{D}$ yarn can be regarded as composed of many tiny facets. As soon as all the facets of the yarn surface are determined, the normal of the each facet is determined accordingly, then the shading effect of the yarn will be calculated according to Phong or Goround [1] model, which gives the yarn the photorealistic effects. The key to build the 3D yarn is to design the facets or the vortexes of the facets or control polygons. Figure 1 demonstrates how a cylinder is built by the facets.

Obviously, it is a tedious work to construct an object by determining so many facets one by one. An efficient method must be found. Many surface modeling technologies were applied widely, such as Bezier surface, Bspline surface [2] as shown in Figure 2. For those surfaces, a grid determined by a serial of controlled vertexes in two directions is used to describe the surface. To accelerate the designing process, software such as OpenGL [3], 3D MAX or DirectX which support B-spline surface or Bezier surface to facilitate the 3D model of an object and gives the object photorealistic effects as well.

To a 3D yarn model, the surface of the yarn can be regarded as a closed surface. Therefore, to construct a 3D model for yarn configuration, there are two issues that have to be considered: 1) representation of the central line; 2) description of the cross-section. Peirce [4] proposed a way to describe yarns in woven structure although his works were based on planar calculation. He thought the yarn is uniform in diameter and the cross-section of the yarn can be circular or elliptical. Kemp [5] and Hearle [6] proposed the cross-section of yarn can be of racetrack and lens depended on the structure. Hearle [7] also proposed that the central line of the yarn be cubic polynomial curve according to energy theory. The shape of the cross-section of the yare is suitable to knitted or braided fabric structure. It should be noted that there is still difference between the real yarn and those hypotheses, perhaps a new assumption will be introduced someday.

Based on these hypotheses, many 3D geometrical models of the yarn were setup. And these models will be introduced according to the method to control the shape of the central line or the cross-section. As for whether the cross-section is one of the shape stated above or how to calculate the true path of the central line by the fabric construction, which are beyond the research of the paper. What concerned in the paper are how to describe the shape of the central line of the yarn if some key points (or interpolated points) on the central line are determined and how to describe the special shape of the cross-section if the cross-section is given. Keep in mind that a good 3D model should not only be able to suit all those hypotheses available at present, but also to the ones may be proposed in future.
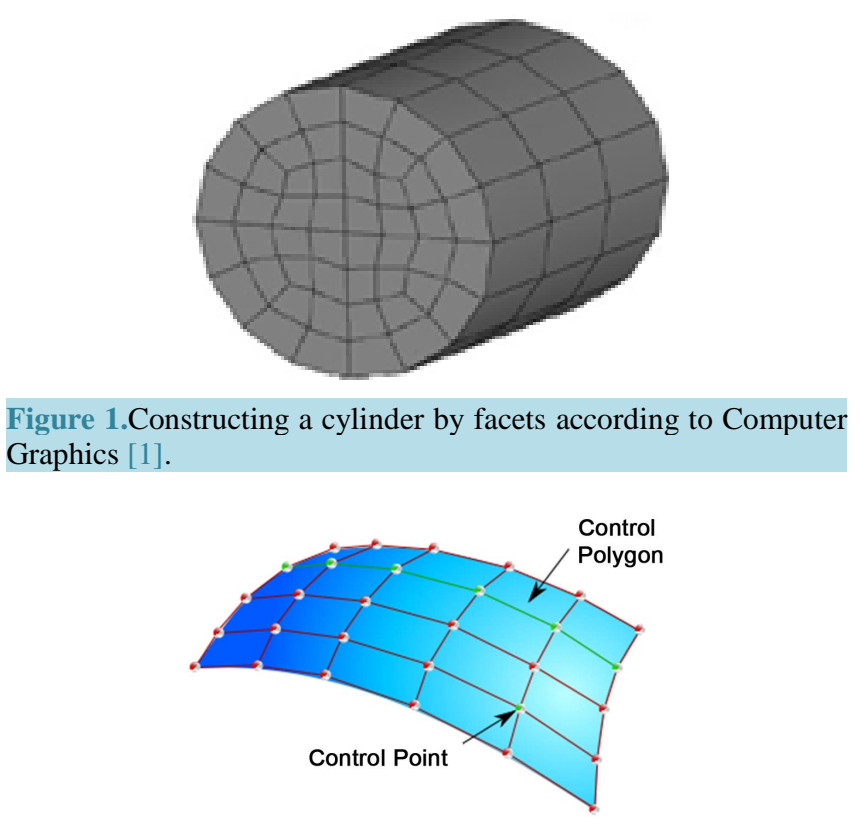

Figure 2. Control points for Bezier/B-spline surface: the shape of the control can be designed by shifting the control point [2]. 


\subsection{Model Based on Sweeping the Cross-Section along the Yarn Path}

Liao and Adanur [8] put up a method to 3D modeling using a computer aided geometric design (CAGD). In this approach, the yarn is assumed to be a uniform cylinder which has a constant cross-section and the center line is an arbitrary spatial curve. The base cross-section is determined first, and it is divided into many arc by several points. A unique plane through any point on the center line and perpendicular to it can be defined. By sweeping the base cross-section curve along the points on the center line, all the points that determine the quadrilateral facets are automatically generated by coordinate transformation. The principle is well explained in Figure 3 . Thus, the two 2D curves (yarn cross-section and yarn central line) are converted into a 3D surface that determines the shape of the object. In the model, the path of the yarn can be the combination of sinusoidal curve and segment line while the cross-section of the yarn can be circular, elliptical and race-track shapes which depend on the construction of the fabric. The structure of 3D woven fabric and braided fabric have been established. However, the cross-section of the yarn is uniform, which is unable to describe the true fabric structure and limits the application.

Lomov [9] proposed a 3D yarn model similar to Liao's model, and software Wise Tex is developed based on Lomov's work. Figure 4 demonstrates Lomov's research. Lin and Long [10] made use of the similar technique to model the yarn comprising of many individual sections of regular shape such as ellipse, lens that can be locally edited to avoid yarns penetrating when used in fabric structure.

\subsection{Model of Uneven Straight Yarn}

In order to reflect the effects of the uneven yarn, $\mathrm{Li}$ [11] builds a yarn which is made up with circles which has different diameters. The diameters can be acquired from the yarn uniformity tester. Segmentation on the surface of the yarn will be carried out by the evenly division of each circle along the center line of yarn. Capitalized on the number of the segment peaks, the contour of the yarn can be obtained by the list of the surface, edge and peak. This method is suitable to straight yarn and it can well reflect the variation of the cross-section. Figure 5 shows the 3D yarn contour in this method. However, the model can only show the straight yarn and cannot be applied to the yarn in fabric structure.
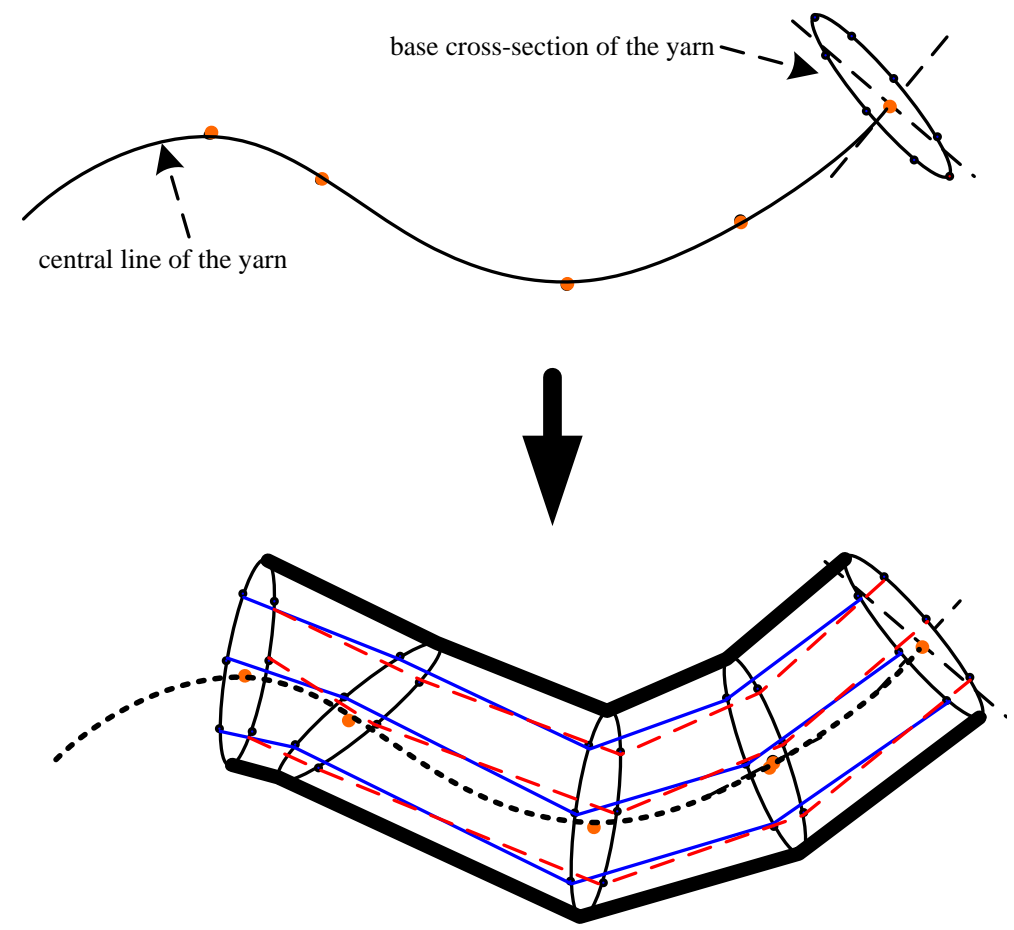

Figure 3. Principle of Liao's yarn model by sweeping the cross-sections along the yarn path [8]. Each cross-section of the yarn is perpendicular to the center line. All the points controlled the quadrilateral facets are automatically generated. 


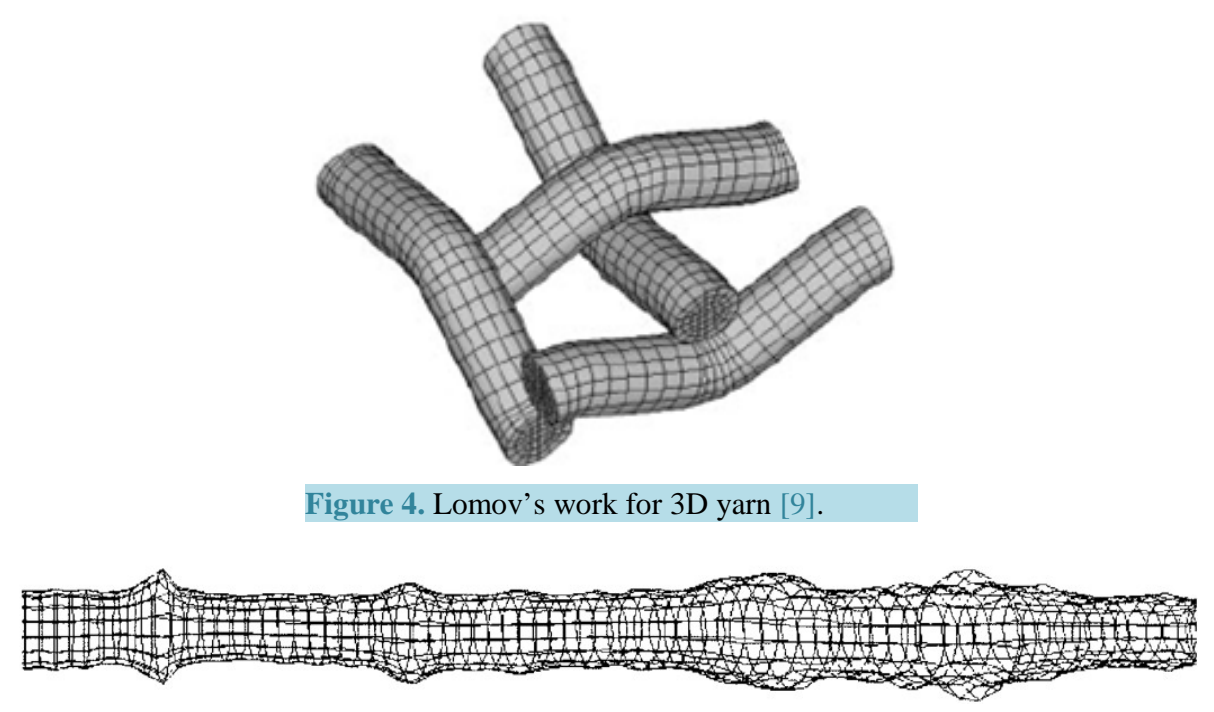

Figure 5. Li’s yarn model with various cross-sections along the central line [10].

\subsection{Based on Polynomial Curve}

Much work has been done to represent the different shapes of the yarn for the changing of shapes varies due to the flattening and strain. Gong and Ozgen [12] [13] acquired the fabric images through ESRF (European Synchrotron Radiation Facility) without disturbing the yarn and fabric structure. The contours of the yarn in the woven fabrics were accurately measured. They confirmed the non-circular nature of yarn cross-sections and also the flattening of yarns at the warp-weft intersections. Two polynomial functions were used to describe the shape of the varied cross-section. The cross-section shape which was modeled by elliptical and polynomial curves was variable along the yarn path. Figure 6 is the 3D representation in this method. Unfortunately, Gong didn't give a real 3D picture to demonstrate his research.

\subsection{Based on Bezier Curve}

Similar to Liao's method, Zhang [14] proposed that the central line of the yarn can be constructed by Bezier curve. The cross-section of the yarn is circular. First, a serial of interpolating points on the central line of the yarn are calculated or given. The control points determining the Bezier curve (central line) are reversely calculated by the interpolating points. Then, applying the sweeping technique, the vertexes of the control grid that determine the Bezier surface are calculated. Different from quadrilateral facets technology, the surface composes of many triangles which ensure the 3 vertexes of any triangle are at the same plane. The triangulation of the Bezier surface will be conducted automatically by OpenGL. The images of 3D yarn can be rendered by Computer Graphics principle.

\subsection{Based on Spline Curve and Super Ellipse}

Jiang and Chen [15] proposed an approach of super ellipse which can design the cross-section in arbitrarily shape. At the same time, the central line of the yarn is represented as spline curve that can pass the given control points. The shape of the cross-section can be designed manually, which makes it convenient to change the yarn shape. The uneven yarn can be modeled and bent. The algorithm can be used in constructing the fabric structure. The software Tex Gen is based on Chen's work.

\subsection{Based on B-Spline Curve}

Nowadays, using B-spline surface is the most popular method to model the configuration of 3D yarn. B-spline curve technology gives the designer more convenience to model the shape of the yarn accurately. Lin and Newton [16] used the cubic B-spline curve to generate the yarn paths, and circle as the form of cross-section. Recently, and Sherburn [17] are able to design irregular yarn using B-spline curve technology. Like Bezier 


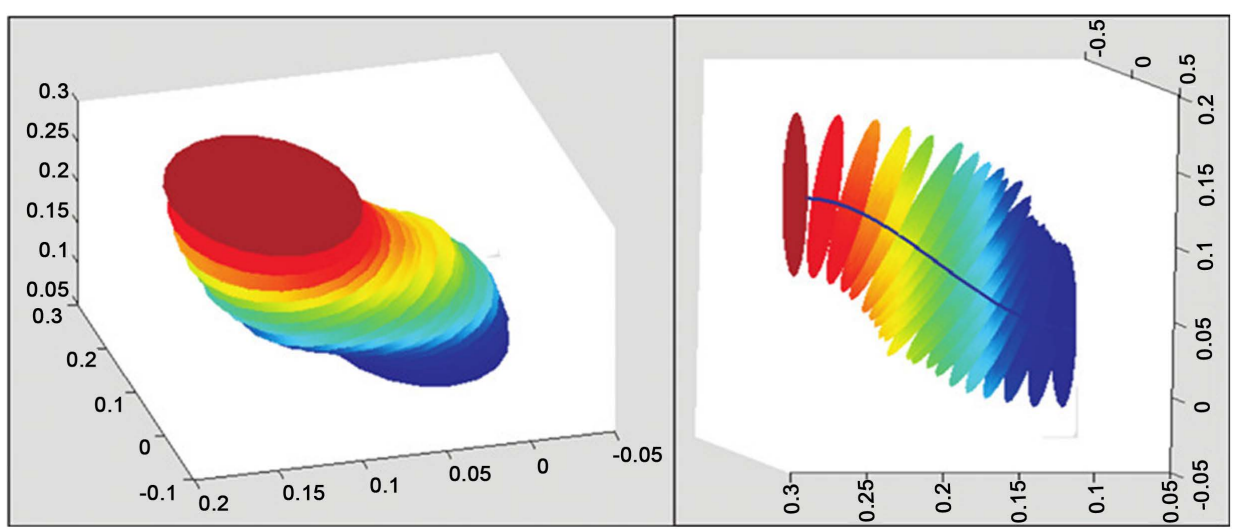

Figure 6. Gong's yarn representation in plain woven fabric [11].

curve, the control points have to be calculated reversely by interpolating points. Unlike Bezier curve, the control points are dependent on the initial conditions, which also causes no uniqueness in control the shape of the yarn. If there is a tiny difference in initial conditions, for example, the positions of the end points change, there may be a big difference in the shape of the curve generated.

Zheng [18] [19] proposed an algorithm that the yarn can be modeled by NURBS (Non Uniform Rational B-Spline Curve) to keep a stable shape of the yarn. The shape preserve quasi uniform cubic B-spline curve is applied to fit the contour of the yarn path accurately, smoothly and stably. Two more adjacent points are inserted before and after a interpolating point respectively according to the lending direction of the all interpolating points, which ensures that the curve go through all the interpolating points. Therefore, the complicated reversely calculation is avoided in determining the control points. In Zheng's research, the quasi uniform quadratic B-spline curve is applied to approximately design the cross-section of a single yarn. The shape of the cross-section can be controlled by a 16-polygon formed by 18 control points. The shape of circle, ellipse, racetrack, lens, bowl, round rectangle can be designed along the yarn path simultaneously (see Figure 7). As long as the base cross-sections at given position are designed, the other cross-sections will be calculated automatically and smoothly. Each cross-section will be put perpendicular to central path. OpenGL is used to design NURBS surface and automatically generate the triangle facetsand give its shading effect. Moreover, some fancy yarns can also be designed by using different cross-section along the path, which will be introduced in the latter part.

Arif Kurbak [20] [21] also built 3D yarn model with 3DS-MAX for knitted fabric. The yarn paths were calculated according to the knitted structure. The cross-section of the yarn is set as circle, therefore, the yarn is actually a cylinder. The loop and tuck heads were taken as ellipses in two dimensions while the rest of the loops and tucks were taken as parabolic helices wrapped on circular cylinders. Thus, the path of yarn in different kind of knitted fabric can be clearly revealed.

To sum up, Table 1 gives a brief summary of characteristics of all the models introduced above.

\section{Sense of Reality}

Although realistic rendering technology provided by OpenGL can draw a yarn with shade effect as shown in Figure 8, it is far from true yarn yet. As it is known to all that the yarn has the twist and hairiness.

Here are some woven models from famous fabric CAD company such as Scot Weave [22], EAT [23] and Tex Gen [24] in Figure 9.

\subsection{Twist}

Zheng [25] added 1D and 2D texture on the surface of yarn to simulate the twist of yarn. For 1D texture as shown in Figure 10(A), parallel lines are drawn on the surface of the yarn with the help of OpenGL. The slop of the lines is dependent on the twist angle of the yarn. The lines are uniformly distributed and the twist effect will be worsen when magnify the yarn. For 2D texture mapping technology as shown in Figure 10(B), an image or a picture scanned from the real yarn will be mapped on the surface of the 3D model to reflect the twist and even hairiness. Ma [26] (Figure 10(C)) and NedGraphics Jacquard Software use the similar mapping technology. Obviously, it requires numerous pictures to imitate different twist effects for actual application. 

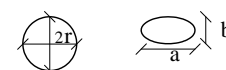

(1)

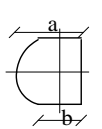

(5)

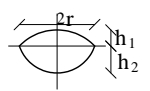

(9)

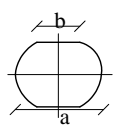

(3)

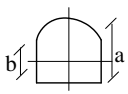

(7)

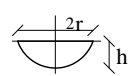

(10)

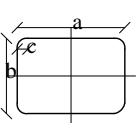

(4)

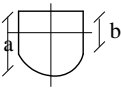

(8)

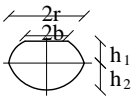

(11)

\section{Figure 7. Cross-sections designable in Zheng's approach [18].}

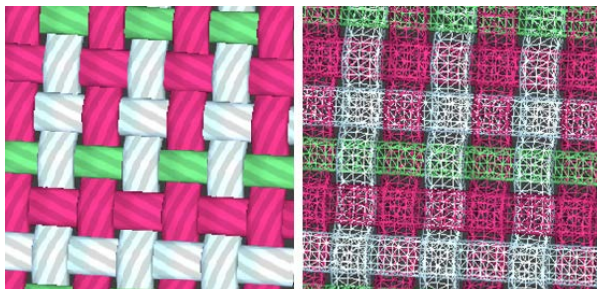

(A)

(B)

Figure 8. (A) 3D plain woven structure with 1D twist effect; (B)

The triangle facets auto-generated by OpenGL.

Table 1. The characteristics of the various methods.

\begin{tabular}{|c|c|c|c|c|c|}
\hline Proposer & Method & Cross-section & Central line & Characteristics & $\begin{array}{c}\text { Implementing } \\
\text { platform }\end{array}$ \\
\hline Liao, Adanur & $\begin{array}{l}\text { Sweeping the } \\
\text { cross-section along } \\
\text { the yarn path }\end{array}$ & $\begin{array}{l}\text { Uniform ellipse, } \\
\text { circle or race-track }\end{array}$ & $\begin{array}{l}\text { Line segment } \\
\text { and arc }\end{array}$ & $\begin{array}{l}\text { Auto-generated } \\
\text { 3D yarn, flexible }\end{array}$ & Unknown \\
\hline $\mathrm{Li}$ & $\begin{array}{l}\text { Assembling } \\
\text { of circles }\end{array}$ & $\begin{array}{c}\text { Circles of different } \\
\text { diameters }\end{array}$ & Straight line & Straight yarn & MSVB \\
\hline Lomov & $\begin{array}{l}\text { Assembling } \\
\text { of solid }\end{array}$ & circle & $\begin{array}{l}\text { Calculated by } \\
\text { WisTex }\end{array}$ & $\begin{array}{l}\text { Uniform cylinder, } \\
\text { flexible }\end{array}$ & MSVC \\
\hline Lin, Long & $\begin{array}{c}\text { Assembling of a } \\
\text { serials of } \\
\text { individual sections }\end{array}$ & $\begin{array}{l}\text { Various cross-sections } \\
\text { of quadratic B-spline } \\
\text { curve, auto-generated } \\
\text { by parameters }\end{array}$ & $\begin{array}{c}\text { Bezier curve, } \\
\text { calculate by TexGen }\end{array}$ & $\begin{array}{l}\text { Shape and size of the } \\
\text { cross-sections can be } \\
\text { changed locally in } \\
\text { fabric structure to } \\
\text { avoid yarn penetrating }\end{array}$ & Python \\
\hline Gong, Azgen & $\begin{array}{l}\text { 3D scanning } \\
\text { of yarn by ESRF }\end{array}$ & Polynomial curve & $\begin{array}{l}\text { Line segment } \\
\text { and arc }\end{array}$ & $\begin{array}{l}\text { The cross-sections } \\
\text { are separated }\end{array}$ & MSVC \\
\hline Zhang & Beizer suface & Beizer curve & Cubic Beizer curve & Uniform cylinder & MSVB, \\
\hline Jiang, Chen & $\begin{array}{l}\text { Spline curve and } \\
\text { super ellipse }\end{array}$ & Uniform Super ellipse & $\begin{array}{l}\text { Cubic spline curve, } \\
\text { calculate by TexEng }\end{array}$ & $\begin{array}{c}\text { The cross-section of the } \\
\text { yarn can be edited, }\end{array}$ & Borland C, \\
\hline Lin, Sherburn & B-spline surface & Various cross-sections & Cubic B-Spline curve & $\begin{array}{l}\text { Auto-generated 3D yarn } \\
\text { including fancy yarn }\end{array}$ & MSVC \\
\hline Zheng & $\begin{array}{l}\text { B-spline surface } \\
\text { or NURBS }\end{array}$ & $\begin{array}{l}\text { Various cross-sections } \\
\text { of quadratic B-spline } \\
\text { curve, auto-generated } \\
\text { by parameters }\end{array}$ & $\begin{array}{l}\text { Cubic shape-preserving } \\
\text { B-spline curve }\end{array}$ & $\begin{array}{l}\text { Auto-generated 3D yarn } \\
\text { including fancy yarn }\end{array}$ & $\begin{array}{l}\text { MSVC, } \\
\text { OpenGL }\end{array}$ \\
\hline Zheng, Zhao & $\begin{array}{c}\text { Assembling of } \\
\text { a serials of } \\
\text { individual sections }\end{array}$ & $\begin{array}{l}\text { C-cardinal spline curve, } \\
\text { arbitrary shape, }\end{array}$ & C-cardinal spline curve & $\begin{array}{l}\text { Cross-section of the yarn } \\
\text { including fancy yarn can } \\
\text { be edited manually }\end{array}$ & $\begin{array}{l}\text { MSVC, } \\
\text { OpenGL }\end{array}$ \\
\hline Arif Kurbak & $\begin{array}{c}\text { Circle and } \\
\text { parabolic helix }\end{array}$ & Circle & $\begin{array}{c}\text { Ellipse or parabolic } \\
\text { helix in } 2 \text { dimensions }\end{array}$ & Circular cylinder & 3DS-MAX \\
\hline
\end{tabular}



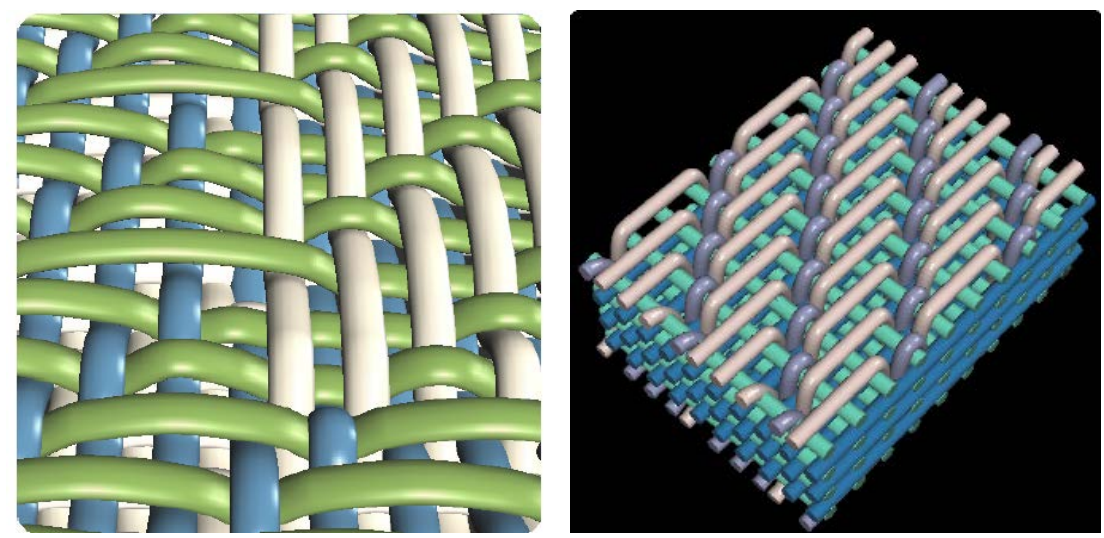

(A)
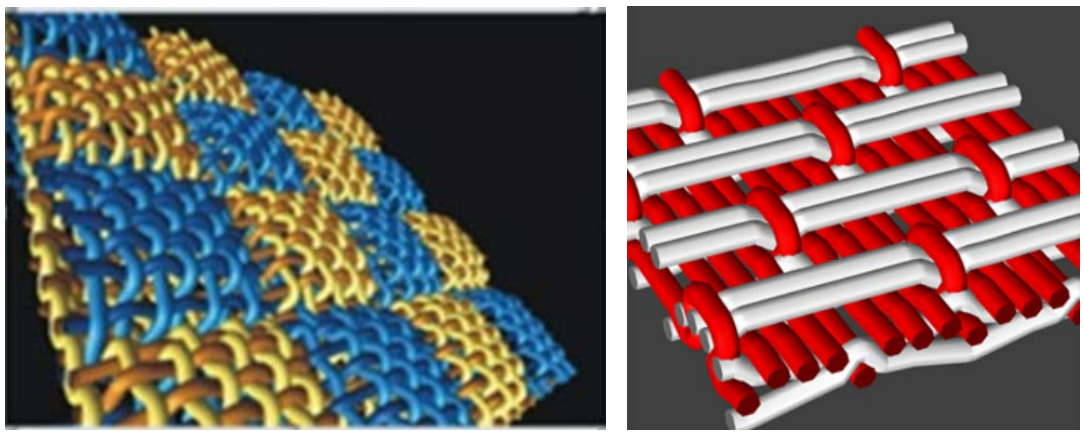

(B)

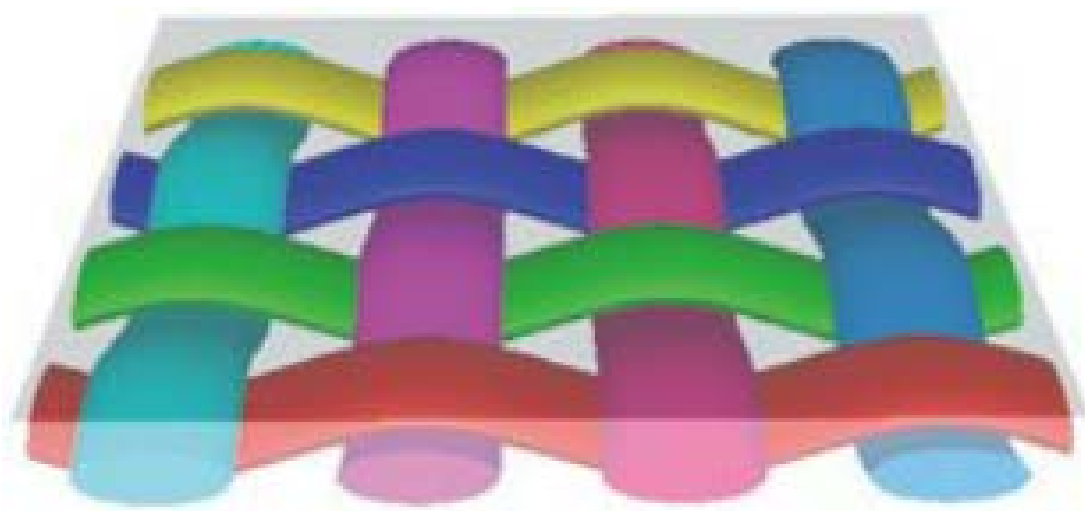

(C)

Figure 9. Fabric Simulation by some companies: (A) Scot Weave [22]; (B) EAT [23] and (C) TexGen [24].

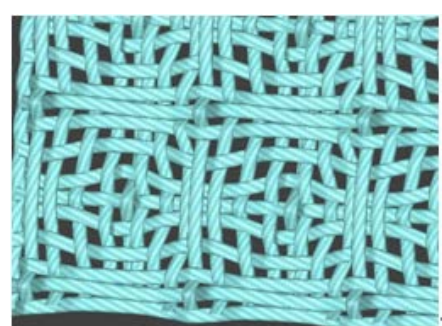

(A)

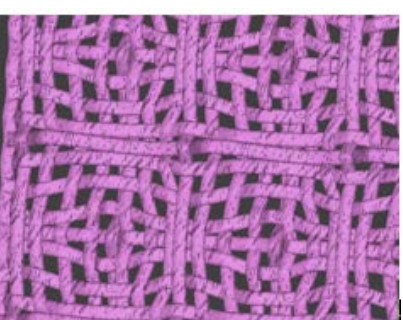

(B)

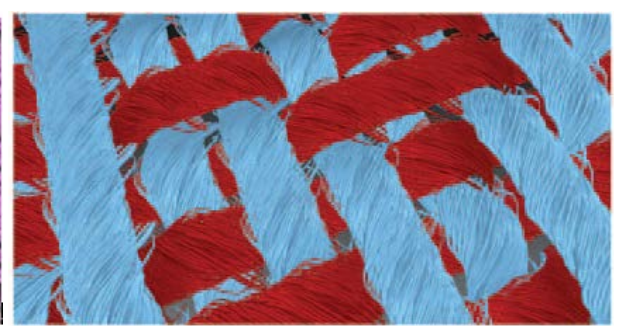

(C)

Figure 10. Zheng's fabric simulating with twist effect (A) 1D texture for twist; (B) 2D texture mapping for twist [25] and (C) is Ma’s study of weave geometries incorporating twisted fiber bundles [26]. 
In order to improve the twist effects, Zheng [19] [27] [28] added bumping texture to acquire more realistic effect or 3D twist effect. By adding troughs and ridges on the contour of the cross-section of the yarn, the surface of the 3D yarn will appear bright and shade effect. When transforming the troughs and ridges at different sections, the twist effect will appear. If a trough or a ridge is deliberately discarded at random, the migration effect of fibers in the structure of the yarn is imitated. The principle is explained in Figure 11 and the imitated image is shown in Figure 12.

Zhao [29] proposed another algorithm to model the yarn based on slice superposition method and C-Cardinal spline curve, which is similar to the shape preserve B-spline curve. The curve passes through the interpolating points except the end points. The drawback can be avoided by repeating the end points two times. Similar to the bumping texture in Zheng [19], the cross-section of the yarn is an irregular polygon as shown in Figure 13(A). By rotating the polygons, a number of polygons are obtained and the series of polygon slices are combined to form a yarn with 3D twist effect as shown in Figure 13(B). The advantages of using the C-Cardinal spline curve are embodied in easy calculation, small memory required. However, the disadvantage is that the curve has not been supported by OpenGL or other software package. If the 3D image is enlarged enough, the slices are separated and the yarn appears discontinuously.

Sriprateep [30] also proposed an algorithm based on fibers' migration in yarns to imitate the 3D twist effect of the ring spun yarn. Each yarn is considered as the assembly of fibers. Each fiber is regarded as a helix along yarn path. The cross-section of the yarn is thought as elliptical shape. Therefore, the yarn can be approximately

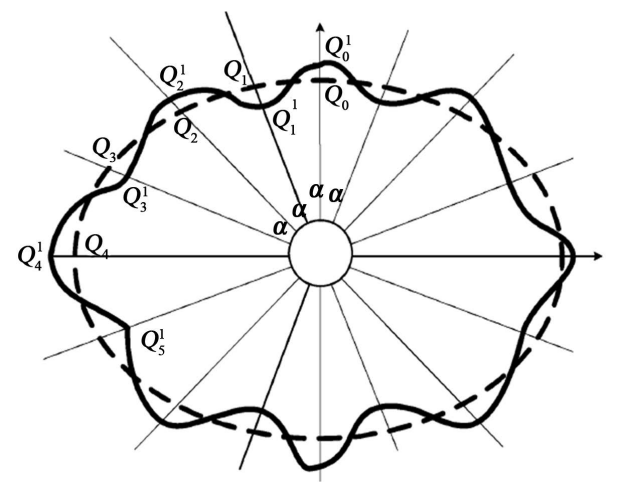

(A)

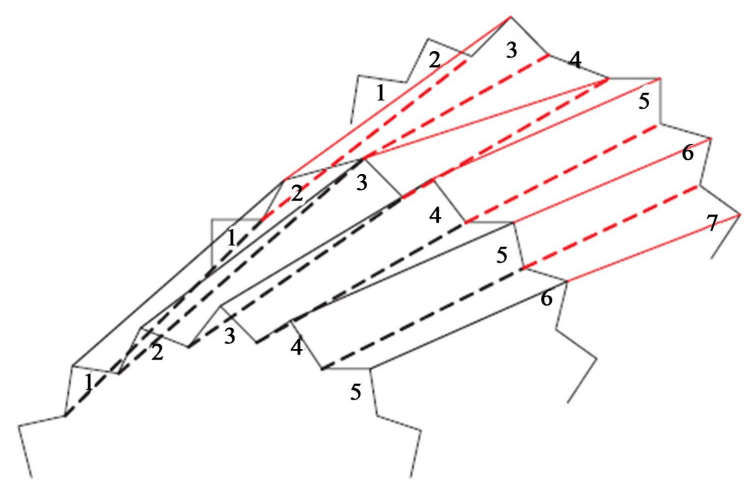

(B)

Figure 11. Zheng added bumping texture as (A) and then (B) the trough and ridge in each cross-section is discarded randomly to create the migration effect of the fiber [19].

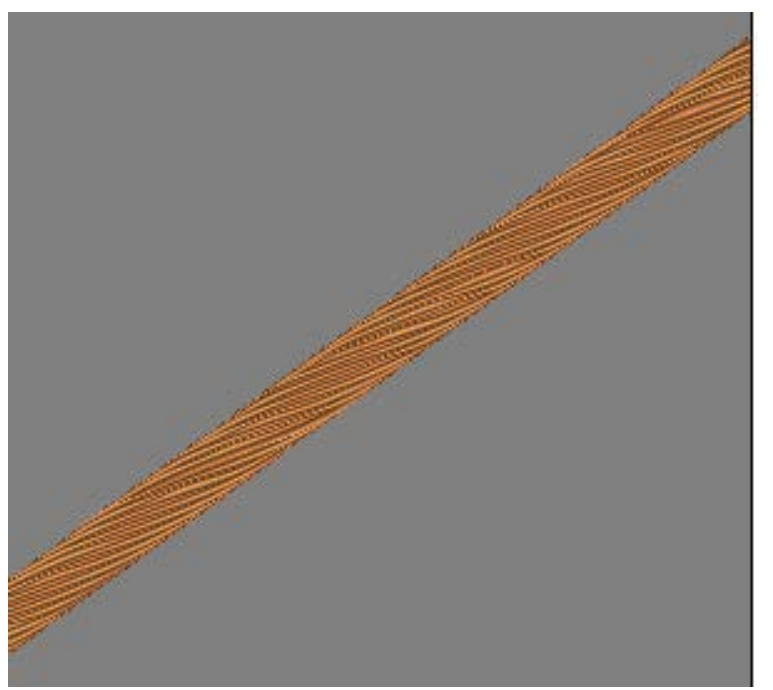

Figure 12. Zheng's yarn model with 3D twist effect in this method [28]. 


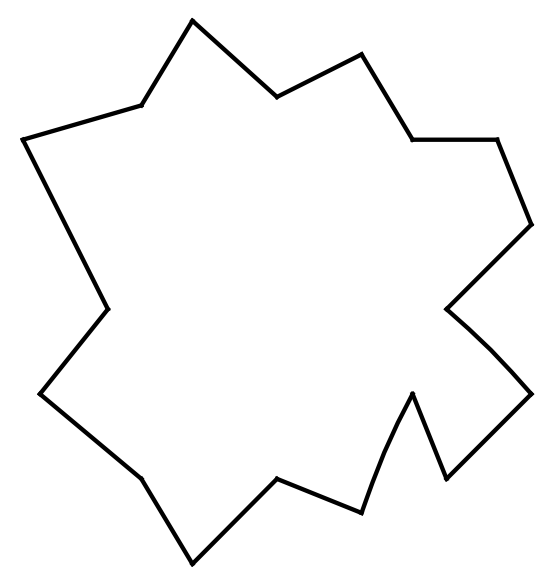

(A)

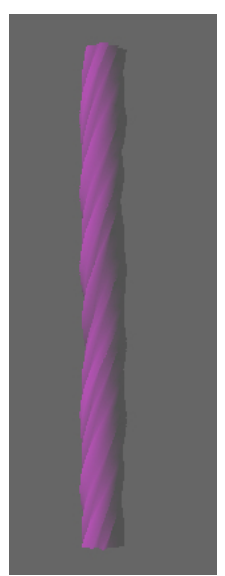

(B)

Figure 13. Zhao using an irregular polygon as cross-section as (A) and (B) is the 3D yarn model with twist effect [29].

constructed by NURBS. The migration parameters include twisting tension, migration period and amplitude, initial phase and frequency of migration pattern. The geometry of yarn structures is eventually modeled by using the Solid Works CAD software package and the model is validated by imitating the process of spinning rayon yarn (see Figure 14). The difference between the algorithm and Zheng's is that Scriprateep uses many NURBS surfaces while Zheng only uses single NUBRS surface to imitate 3D twist effect.

\subsection{Hairiness}

A realistic model for 3D yarn should reflect the hairiness effect. Zhong [31] achieved the aim by using projected triangles to simulate the hairiness, but the effect could be weakened if the 3D image was enlarged. As Figure 15(A) shown, Xu [32] realized a photorealistic effect yarn by rotating an image including many lighten points. The shade effect, twist effect and hairiness effect were demonstrated excellently. However, it is not a real 3D model for yarn as the image generated cannot be rotated or viewed in different direction. Effect in this approach is in Figure 15(B). Zheng [19] proposed that NURBS surface be used to imitate the projected fiber similar to the technique for yarn. The length, projecting angle and the distribution of the hairs can be designed.

\section{Application of 3D Single Yarn}

\subsection{Folded Yarn}

Folded yarn comprises of two or more single yarn, so it can be designed if the central paths of all the component yarns are determined. In Zheng [33], a two-ply or three-ply folded yarn is modeled by rotating two or three circular cross-section around so called folded yarn center, therefore, the central line of each component single yarn is actually a helix. The sizes of the twist are controlled by the screw pitch of the helix while the direction of the twist is determined by the rotating angle. In Zhao and Zheng [34], the cross-section of the single yarn in the folded yarn was assumed to be circular or irregular polygon by slice superposition method which is shown in Figure 16(A). In order to demonstrate the twist effect of the folded yarn, at least 4 slices of cross-sections and their centers of each component yarn will be calculated for every other $90^{\circ}$. Figure 16(B) shows a bent folded yarn based on the algorithm similar to Zheng [22] [28]. If more than 20 single yarns are folded together, the folded yarn appears as a single yarn as each yarn is so slim that looks like a single fiber. Figure 16(C) gives the effects which is similar to Sriprateep's work [30].

\subsection{Fancy Yarn}

It is certain that the fancy yarn can be modeled by 3D technology although it is rare. For fancy yarn, there are varied sections along the yarn path. It can be folded yarn whose components are of various color, thickness or configuration in space. Based on Zheng's [19] [35] [36] algorithm, the cross-sections and configuration of the yarn can be changed based on NURBS, the fancy yarn will be modeled. Figure 17(A) demonstrates a slub yarn 


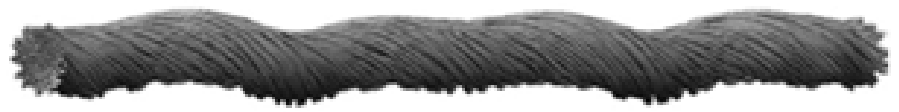

Figure 14. Sriprateep's yarn model with 3D twist effect which based on fiber's migration [30].

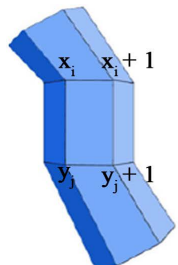

(a)

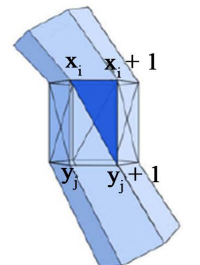

(b)

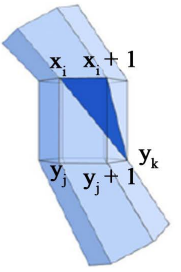

(c)

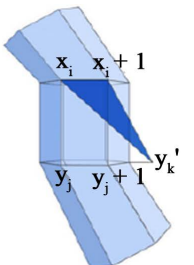

(d)

(A)
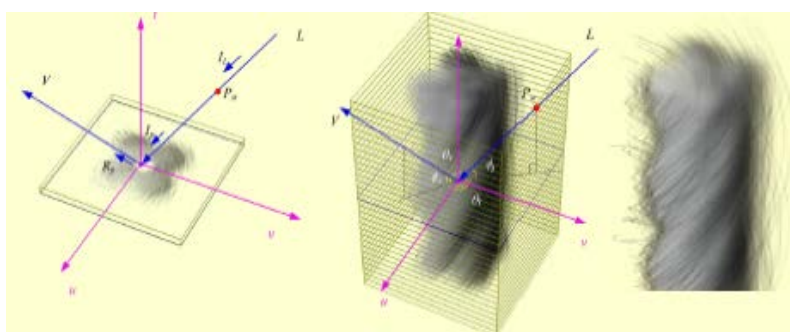

(B)

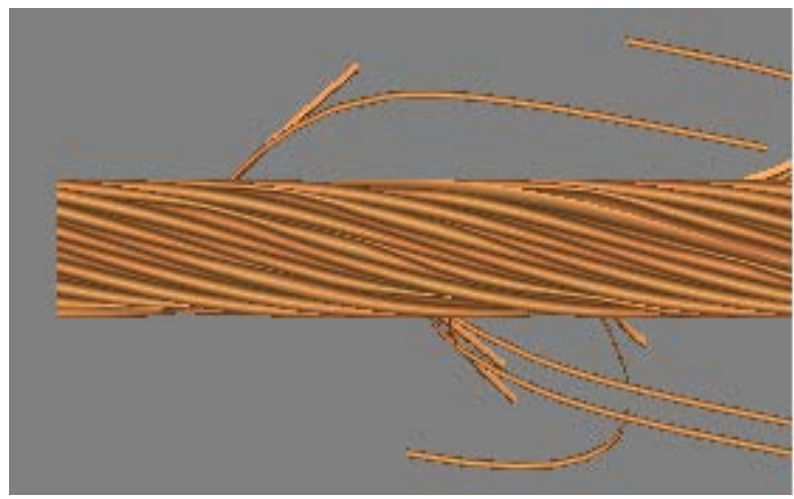

(C)

Figure 15. Hairiness effect in different methods: (A) is Zhong's yarn model with hairiness built by projected triangles [31]; (B) Xu's yarn model with photorealistic effect based on image [32]; (C) Zheng added hairiness by NURBS surface [19].

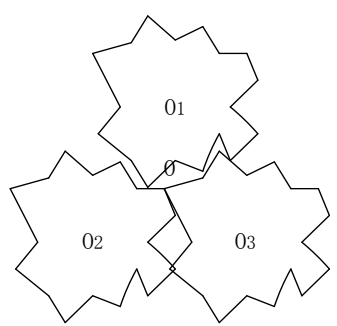

(A)

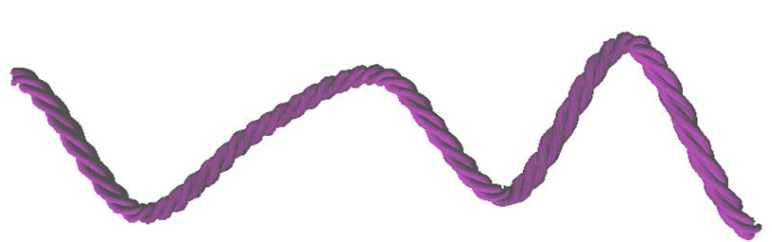

(B)

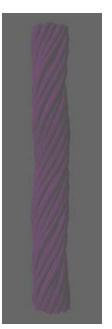

(C)

Figure 16. (A) Irregular polygon in Zhao's yarn model; (B) Zhao's 3D model of folded yarn; (C) A single yarn is imitated by modeling multi-folded yarn [34]. 
modeled on the principle. In fact, loop yarn, knop yarn, slub yarn, snarly yarn can also be designed when the frequency/size of the effect spot, path/color of the component single yarn are designed. Based on C-Cardinal spline curve, Zhao [34] demonstrates a slub yarn as shown as Figure 17(B). The slub yarn includes even parts and uneven parts which change from thinner to the thicker diameter. The twist effect can be improved by increasing the rotating a larger angle of the two neighboring slices at the thinner area.

Zhuge [36] designed the contour of fancy yarn was drawn by Bezier tools. Then template of illumination factor was applied to the model. Then, loop hairiness, end hairiness or floating hairiness can be generated by random noise point.

\subsection{Structure of Fabric}

If the geometric structure in a woven, knitted or braid fabric is calculated, or in other words, the shape and the path of the each yarn in fabric is determined, the 3D geometric structure of the fabric can be imitated. Figure 18(A) shows a plain fabric with the diameter of the yarn changes along the central path; Figure 18(B) shows the same fabric structure with 3D twist effect; Figure 18(C) shows a plain fabric comprised of folded yarn; Figure 19(A) and Figure 19(B) [35] show two fancy woven fabrics respectively; Figure 20 shows a knitted fabric structure.

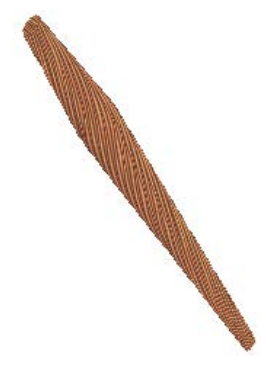

(A)

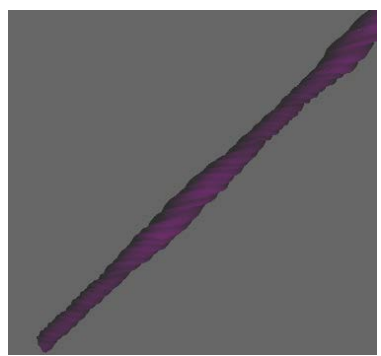

(B)

Figure 17. (A) Zheng's yarn model of section part in slub yarn [19]; (B) Zhao's slub yarn model with different twist in the thicker and thinner area [34].

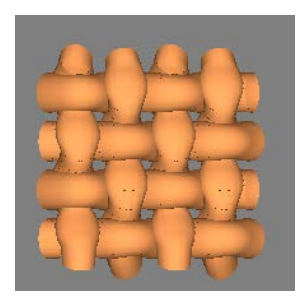

(A)

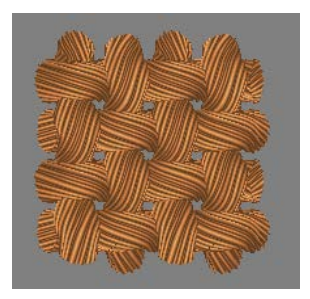

(B)

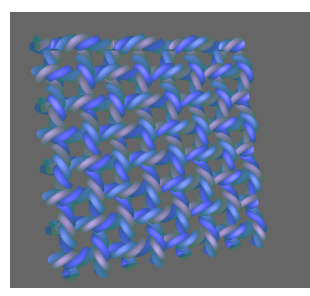

(C)

Figure 18. (A) Plain fabric of various cross-sections without twist; (B) Plain fabric of various cross-sections with twist; (C) Plain fabric comprised of folded yarn.

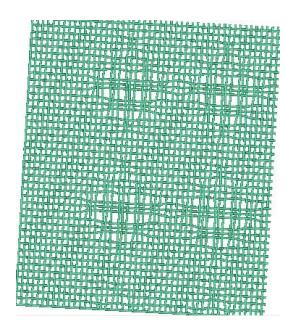

(A)

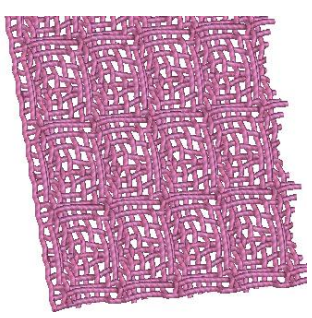

(B)

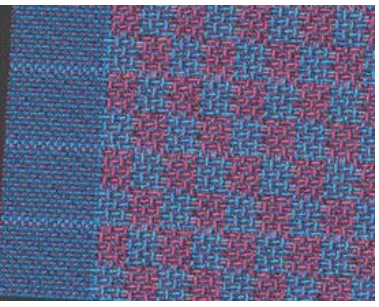

(C)

Figure 19. Structure of fancy weaves (A) Effect of mesh weaves; (B) Honey comb weaves [36]; (C) The structure of interchanging double cloth. 


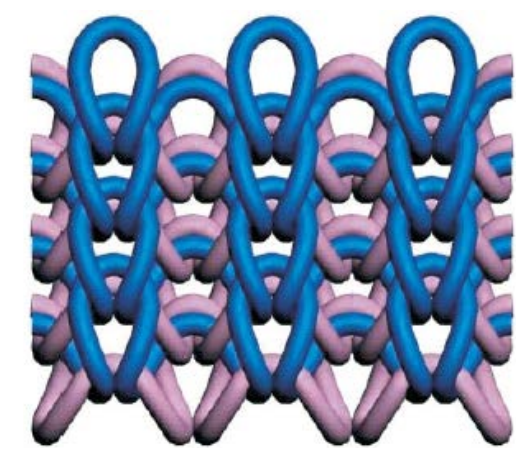

Figure 20. Structure of knitted fabric in Kayacan’s study [37].

\section{Conclusion}

Multiple approaches have been reviewed for modeling 3D yarn geometric configuration. The yarns are shaped on the theory of Peirce while the 3D constructing technology is based on Computer Graphics. And all the methods are related with designing the cross-sections along the yarn path. Comparatively speaking, the method based on spline curve is the most popular one since it gives the most flexibility in designing not only non-uniform single yarn with different cross-sections and twist effect, but also folded yarn, fancy yarn including slub yarn, knep yarn. The yarn can bent or deform in 3D space freely so it can be used in woven fabric's structure conveniently. The model can also be applied to the structure of the knitted fabric, braided fabric so long as the central line of the yarn is given.

\section{Acknowledgements}

Authors would like to thank Henan Provincial Collaborative Innovation Center for Textiles and Appeals, Zhengzhou Municipal Bureau of Science and Technology for supporting the research.

\section{References}

[1] Hearn, D. and Baker, M.P. (1998) Computer Graphics (C Version). Prentice Hall, Inc., Upper Saddle River, 335-355.

[2] Pigel, L. and Tiller, W. (1996) The NURBS Book. 2nd Edition, Springer Press, Berlin.

[3] Angel, E. (2003) Interactive Computer Graphics: A Top-Down Approach with OpenGL. 3rd Edition, Person, Addison Wisley, Boston, 193-194.

[4] Peirce, F.T. (1937) Geometry of Cloth Structure. Textile Research Journal, 28, 45-96.

[5] Kemp, A. (1958) An Extension of Peirce's Applicable to the Treatment of Non-circular Threads. Journal of the Textle Institute, 49, 45.

[6] Hearle, J.W.S. and Shanahan, W.J. (1978) An Energy Method for Calculations in Fabric Mechanics. Part I: Principles of the Method. Journal of the Textle Institute, 69, 81-89. http://dx.doi.org/10.1080/00405007808631425

[7] Shanahan, W.J. and Hearle, J.W.S. (1978) An Energy Method for Calculations in Fabric Mechanics. Part II: Examples of Application of the Method to Woven Fabrics. Journal of the Textle Institute, 69, 81-89. http://dx.doi.org/10.1080/00405007808631426

[8] Liao, T. and Adanur, S. (1998) A Novel Approach to Three-Dimensional Modeling of Interlaced Fabric Structures. Textile Research Journal, 68, 841-847. http://dx.doi.org/10.1177/004051759806801109

[9] Lomov, S.V., Mikolanda, T., Kosek, M. and Verposest, I. (2006) Model of Internal Geometry of Textile Fabrics: Data Structure and Virtual Reality Implementation. Journal of the Textile Institute, 98, 1-13. http://dx.doi.org/10.1533/joti.2006.0251

[10] Li, Y., Zeng, P. and Zhao, L. (2004) The Study on the Clothing Simulation Based on 3D Graphics. Journal of Textile Research (China), 25, 67-69.

[11] Lin, H., Zeng, X., Martin, S., Andrew, L. and Mike, C. (2011) Automated Geometric Modelling of Textile Structures. Textile Research Journal, 82, 1689-1702.

[12] Gong, R.H., Ozgen, B. and Soleimani, M. (2009) Modeling of Yarn Cross-Section in Plain Woven Fabric. Textile Research Journal, 79, 1014-1020. http://dx.doi.org/10.1177/0040517508101799 
[13] Ozgen, B. and Gong, R.H. (2011) Modelling of Yarn Flattening in Woven Fabrics. Textile Research Journal, 81, 1523-1531.

[14] Zhang, R., Huang, X. and Li, R. (2005) 3D Computer Simulating of Weave Fabric. Journal of Textile Research (China), 26, 42-44.

[15] Jiang, Y. and Chen, X. (2004) The Structural Design of Software for Simulation and Analysis of Woven Fabric by Geometry Model. Textile Institute World Conference (China), Donghua University Press, Shanghai, 1298-1301.

[16] Lin, H.Y. and Newton, A. (1999) Computer Representation of Woven Fabric by Using B-Splines. Journal of the Textile Institute, 90, 59-72. http://dx.doi.org/10.1080/00405009908658691

[17] Sherburn, M. (2007) Geometric and Mechanical Modeling of Textiles. Ph.D. Thesis, Thesis Submitted to The University of Nottingham for the Degree of Doctor of Philosophy.

[18] Zheng, T.Y. and Cui, S. (2006) The Study on Constructing the 3D Yarn Model by B-Spline Surface. Part I: Construction of 3D Yarn Model with Different Cross Sections. Journal of Textile Research (China), 27, 53-57.

[19] Zheng, T.Y. and Cui, S. (2007) Improvement on Modeling a 3D Yarn with B-Spline Surface. Journal of Textile Research, 28, 35-44.

[20] Kurbak, A. and Soydan, A.S. (2009) Geometrical Models for Balanced Rid Knitted Fabrics Part III: $2 \times 2$, $3 \times 3$, $4 \times 4$, and $5 \times 5$ Rib Fabrics. Textile Research Journal, 79, 618-625. http://dx.doi.org/10.1177/0040517508096218

[21] Kurbak, A. and Soydan, A.S. (2009) Geometrical Models for Cardigan Structures. Part I: Full Cardigan. Textile Research Journal, 79, 1281-1300. http://dx.doi.org/10.1177/0040517508102258

[22] http://scotweave.com/products/technical-weaver/

[23] http://www.designscopecompany.com/cross-section/

[24] http://www.bluefox.com

[25] Zheng, T.Y. and Huang, G. (2002) The Setup of 3D Model for Fabric Yarns (China). Journal of Textile Research, 23, 13-15.

[26] Ma, L., George, B., Hu, J. and Zhang, J. (2011) A Novel Weave Pattern Encoding Method Using Neighbor Information and Its Applications. Textile Research Journal, 81, 632-648.

[27] Zheng, T. and Cui, S. (2006) The Study on Constructing the 3D Yarn Model by B-Spline Surface. Part II: Inserting 3D Bumping Texture on the Surface of the Yarn. Journal of Textile Research (China), 27, 53-57.

[28] Zheng, T.Y. (2010) Study on General Geometrical Modeling of Single Yarn with 3D Twist Effect. Textile Research Journal, 80, 867-879.

[29] Zhao, Z., Zheng, T.Y., Wu, Z. and Li, T. (2014) Study on Constructing the Model of Ply Yarn by the Cubic C-Cardinal Spline Cure. Journal of Textile Research, in press.

[30] Sriprateep, K. and Bohez, E.L.J. (2009) A New Computer Geometric Modelling Approach of Yarn Structures for the Conventional Ring Spinning Process. Journal of Textile Institute, 100, 223-236. http://dx.doi.org/10.1080/00405000701757958

[31] Zhong, H., Xu, Y., Guo, B. and Shum, H. (2001) Realistic and Efficient Rendering of Free-Form Knitwear. The Journal of Visualization and Computer Animation (China), 12, 13-22. http://dx.doi.org/10.1002/vis.241

[32] Xu, Y. (2000) Photorealistic Rendering of Knitwear Using the Lumislice. Institute of Software, Academia China.

[33] Zheng, T.Y. (2001) A 3D Model for Folded Yarns. Journal of Textile Research (China), 23, 20-21.

[34] Zhao, Z. (2014) Three-D Simulation Research of Ply Yarn Based on the Cubic C-Cardinal Spline Cure Modeling Techniques. Master's Thesis, Zhongyuan University of Technology, Zhengzhou.

[35] Zheng, T.Y. (2005) 3D Appearance Imitation of Fancy Weaves. Journal of Textile Research (China), 26, 35-38.

[36] Zhuge, Z. and Zhang, F. (2005) Numerical Simulation of Fancy Yarn. Journal of Zhejiang University (China), 39, 1529-1536.

[37] Kayacan, O. and Kurbak, A. (2008) Basic Studies for Modeling Complex Weft Knitted Fabric Structures Part IV: Geometrical Modeling of Miss Stitches. Textile Research Journal, 78, 659-663.

http://dx.doi.org/10.1177/0040517507087663 